\title{
Constituintes químicos das folhas e caule de Croton sellowii (Euphorbiaceae)
}

\author{
Sebastião F. Palmeira Júnior ${ }^{l}$, Vanessa L. Alves ${ }^{l}$, Fabyanne S. Moural, Larissa F.A. Vieira ${ }^{l}$, \\ Lucia M. Conserva ${ }^{1 *}$, Rosangela P.L. Lemos ${ }^{2}$ \\ ${ }^{1}$ Departamento de Química, Universidade Federal de Alagoas, 57072-970, Maceió, AL, Brasil, \\ ${ }^{2}$ Instituto do Meio Ambiente do Estado de Alagoas, 57017-320, Maceió-AL, Brasil
}

\begin{abstract}
RESUMO: O presente trabalho descreve o isolamento de terpenóides (sitosterol, estigmasterol,

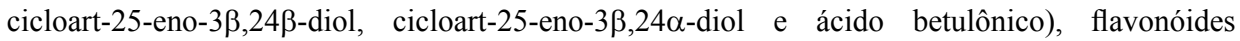
(artemetina, crisosplenetina, casticina, penduletina e tilirosídeo) e fenilpropanóide (treo-7-etoxi-8hidroxi-diidroanol) nas folhas e caule de Croton sellowii Baill. Todos os compostos isolados estão sendo descritos pela primeira vez nesta espécie e foram identificados com base na análise de dados espectrais (IV, UV e RMN, incluindo APT, DEPT, COSY, NOESY, HMQC e HMBC), bem como pela comparação com dados descritos na literatura.
\end{abstract}

Unitermos: Croton sellowii, Euphorbiaceae, flavonóides, terpenóides, fenilpropanóide.

\begin{abstract}
Chemical constituents from the leaves and stems of Croton sellowii (Euphorbiaceae)". This work describes the isolation of terpenoids (sitosterol, stigmasterol,

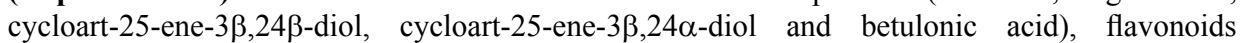
(artemetin, crysosplenetin, casticin, penduletin and tiliroside) and phenylpropanoid (threo-7ethoxy-8-hydroxy-dihydroanol) from the leaves and stems of Croton sellowii Baill. All isolated compounds are being described for the first time in this species and were identified on basis of the spectral data (IR, UV, and NMR, including APT, DEPT, COSY, NOESY, HMQC and HMBC), and by comparison with literature data.
\end{abstract}

Keywords: Croton sellowii, Euphorbiaceae, flavonoids, terpenoids, phenylpropanoid.

\section{INTRODUÇ̃̃̃O}

O gênero Croton, o segundo mais numeroso da família Euphorbiaceae (Farnsworth et al., 1969), compreende cerca de 700 a 800 espécies de distribuição neotropical, com poucos representantes paleotropicais (Randau et al., 2004), das quais cerca de 300 ocorrem no Brasil (Schultz, 1984). Muitas dessas espécies são utilizadas na medicina popular para os mais variados fins (Farnsworth et al., 1969; Kumar et al., 1980; Hedberg et al., 1983; Macedo; Ferreira, 2004). Dentre as atividades farmacológicas experimentalmente comprovadas para o gênero Croton colocam em destaque o seu potencial antiinflamatório (Falcão et al., 2005), antiulcerogênico (Almeida et al., 2002), antidiabético (Barbosa-Filho et al., 2005), inibidores da enzima acetilcolinesterase (Barbosa-Filho et al., 2006) entre outras (Pieters et al., 1992; Piacente et al., 1998; Otshudi et al., 2000; Palmeira Júnior, 2005).

A química do gênero Croton tem sido bastante explorada e os estudos fitoquímicos efetuados têm conduzido ao isolamento de alcalóides (Farnsworth et al., 1969; Pieters et al., 1995; Bittner et al., 1997), flavonóides, triterpenóides e uma grande variedade estrutural de diterpenóides (Farnsworth et al., 1969; Lencina et al.,
2001; Santos et al., 2005; Palmeira Júnior, 2005). Além dessas classes, lignanas (Pieters et al., 1992; 1993; 1995), benzenóides (Cai et al., 1993; Chen et al., 1994; Bittner et al., 1997), poliprenóides (Hernandez; Delgado, 1992) e metais (Macari et al., 2002) também foram encontrados. Muitas das espécies são produtoras de óleo essencial cuja composição química é rica em mono- e sesquiterpenóides e fenilpropanóides (Farnsworth et al., 1969; Craveiro et al., 1990; Lemos et al., 1992).

Croton sellowii Baill é um arbusto cujo extrato em acetona das raízes apresenta atividade antibacteriana (Farnsworth et al., 1969). A literatura relata nas raízes desta espécie a ocorrência do antibiótico selovicina e sua atividade antibacteriana (Leôncio d'Albuquerque et al., 1968; Lewis et al., 1977), bem como a identificação através de CG-EM de sesquiterpenos, $n$-alcanos, ésteres, álcoois e alquilbenzenos nas folhas, raízes e caule (Palmeira Júnior et al., 2004). O presente trabalho relata a primeira ocorrência de terpenóides (1-5), flavonóides $(6-9,11)$ e fenilpropanóide (10) nas folhas e caule desta espécie.

\section{MATERIAL E MÉTODOS}

\section{Métodos gerais}


As cromatografias em coluna foram efetuadas em gel de sílica (70-230 e 230-400 mesh, Merck) e em Sephadex LH-20 (Pharmacia). Os espectros UV-VIS foram registrados, em metanol, em espectrofotômetro Hitachi, modelo U-3000. Os espectros IV foram registrados em pastilhas de $\mathrm{KBr}$ utilizando um espectrofotômetro PerkinElmer, FT-IR 1750. Os espectros de RMN ( ${ }^{1} \mathrm{H}: 200,300$ e $500 \mathrm{MHz} ;{ }^{13} \mathrm{C}: 50,75$ e $125 \mathrm{MHz}$ ) foram obtidos em espectrômetros Mercury-Varian 200, Brucker DPX-300 e DRX-500, respectivamente. O sinal residual do solvente ou o TMS foram utilizados como referência interna.

\section{Material vegetal}

As folhas e o caule de um espécime de $C$. sellowii foram coletados, em novembro de 1999, na Área de Proteção Ambiental de Santa Rita (Mucuri), município de Marechal Deodoro, Alagoas, Brasil. A identificação botânica da espécie foi efetuada por Rosangela Pereira de Lyra Lemos do Departamento de Botânica do Instituto do Meio Ambiente do Estado de Alagoas (IMA/AL), onde uma exsicata foi depositada (MAC-10750).

\section{Extração e isolamento dos constituintes químicos}

As folhas (1500 g) e o caule (1900 g) de C. sellowii, após secagem a temperatura ambiente e trituração, foram extraídos através de maceração com acetona seguido de EtOH 90\%. Após remoção dos solventes em evaporador rotatório, os extratos obtidos [acetona: folhas (139 g) e caule (47 g); EtOH: folhas (126 g) e caule $(31 \mathrm{~g})$ ] foram suspensos em solução de $\mathrm{MeOH}-$ $\mathrm{H}_{2} \mathrm{O}(3: 2)$ e extraídos sucessivamente com $\mathrm{C}_{6} \mathrm{H}_{14}, \mathrm{CHCl}_{3}$ e AcOEt. Os extratos em $\mathrm{C}_{6} \mathrm{H}_{14}$ oriundos das folhas, após análise comparativa através de cromatografia em camada delgada, foram reunidos (72 g) e tratados com solução aquosa de $\mathrm{NaOH} 4 \%$ e, posteriormente, extraído com $\mathrm{C}_{6} \mathrm{H}_{14}$. A porção neutra $(30 \mathrm{~g})$, após fracionamento cromatográfico em gel de sílica (70-230 e 230-400 mesh, misturas de $\mathrm{C}_{6} \mathrm{H}_{14}$-AcOEt em gradiente crescente de polaridade), Sephadex LH-20 com $\mathrm{MeOH}$ e sucessivas recristalizações com acetona forneceu uma mistura, M1, contendo sitosterol e estigmasterol $(\mathbf{1}+\mathbf{2}, 72 \mathrm{mg})$, ácido betulônico $(\mathbf{3}, 17 \mathrm{mg})$ e uma mistura, $\mathrm{M} 2$, contendo os epímeros cicloart-25-eno-3 $\beta, 24 \alpha$-diol e cicloart-25-eno$3 \beta, 24 \beta$-diol $(\mathbf{4}+\mathbf{5}, 26 \mathrm{mg})$.

Os extratos em $\mathrm{CHCl}_{3}$ (folhas: $4 \mathrm{~g}$; caule: $33 \mathrm{~g}$ ) foram fracionados em gel de sílica (70-230 mesh) com misturas de $\mathrm{C}_{6} \mathrm{H}_{14}$-AcOEt em proporções crescentes de polaridade. As frações provenientes das folhas, após permeação em gel (Sephadex LH-20 com $\mathrm{MeOH}$ ) e CCD preparativa [gel de sílica $\mathrm{PF}_{254}, \mathrm{CHCl}_{3}$-AcOEt (7:3)], forneceram artemetina $(6,40 \mathrm{mg})$, crisosplenetina $(7,12$ $\mathrm{mg}$ ), uma mistura, M3, contendo casticina e penduletina $(\mathbf{8}+9,30 \mathrm{mg})$ e treo-7-etoxi-8-hidroxi-diidroanol (10, $40 \mathrm{mg}$ ). Por sua vez, as frações oriundas do caule, após permeação em gel (Sephadex LH-20 com MeOH) e
CCD preparativa [gel de sílica $\mathrm{PF}_{254}, \mathrm{C}_{6} \mathrm{H}_{6}$-AcOEt (8:2)], conduziram ao isolamento de artemetina $(6,75 \mathrm{mg})$ e crisosplenetina $(7,21 \mathrm{mg})$.

O extrato em AcOEt (2,8 g), oriundo da partição do extrato em etanol do caule, foi fracionado em gel de sílica (70-230 mesh, $\mathrm{C}_{6} \mathrm{H}_{14}$-AcOEt 1:1). As frações obtidas após análise comparativa através de CCD em diferentes sistemas de eluentes foram agrupadas. $\mathrm{O}$ material da subfração $30-65(0,53 \mathrm{~g})$ após sucessivos fracionamentos cromatográficos em gel de sílica (230-400 mesh, em misturas de $\mathrm{C}_{6} \mathrm{H}_{14}$-AcOEt) e CCD preparativa (gel de sílica $\mathrm{PF}_{254}, \mathrm{C}_{6} \mathrm{H}_{6}$-AcOEt 1:1) conduziu ao isolamento de artemetina $(\mathbf{6}, 10 \mathrm{mg})$ e tilirosídeo $(\mathbf{1 1}, 30$ $\mathrm{mg})$.

As substâncias isoladas tiveram suas estruturas identificadas com base na análise dos dados espectrais (IV, UV e RMN, incluindo APT, DEPT, COSY, NOESY, HMQC e HMBC), bem como pela comparação com dados descritos na literatura.

\section{RESULTADOS E DISCUSSÃO}

As substâncias 1 (Macari et al., 1990), 2 (Marques, 2001) e 3 (Carpenter et al., 1980; Mahato; Kundu, 1994) foram identificadas pela comparação dos dados espectrais obtidos (IV e RMN) com os descritos na literatura.

A mistura M2 foi identificada como sendo constituída pelos epímeros em C-24 do triterpeno cicloart25-eno-3 $\beta, 24$-diol $(\mathbf{4}+5)$, na proporção de $1: 3$, tendo como base à análise dos espectros de RMN, incluindo HMBC, bem como pela comparação com os dados de um dos isômeros descritos na literatura (Anjaneyulu et al., 1993). A diferença de intensidade relativa de sinais duplicados no espectro de $\mathrm{RMN}{ }^{13} \mathrm{C}$ definiu a proporção dos componentes na mistura. $\mathrm{O}$ espectro de $\mathrm{RMN}{ }^{1} \mathrm{H}$ a $500 \mathrm{MHz}$ mostrou sinais para hidrogênios carbinólicos [ $\delta$ 3,29 (m, H-3) e 4,04 (m, H-24)], ligação dupla do tipo metileno terminal $[\delta$ 4,86 (sl, H-26a) e 4,63 (sl, $\mathrm{H}-26 \mathrm{~b})]$, grupos metilas ligados a carbonos $\mathrm{sp}^{3}[\delta 0,83$; 0,$90 ; 0,91 ; 0,99\left(2 \times \mathrm{CH}_{3}\right)(\mathrm{s}$, cada) $)$ e a $\mathrm{sp}^{2}[1,74$ (s, $\mathrm{Me}-27)]$, além dos sinais característicos de hidrogênios do grupo metilênico de anel ciclopropano $[\delta 0,35(d$, $J=4,1 \mathrm{~Hz}, \mathrm{H}-19 \mathrm{a})$ e $0,58(d, J=4,1 \mathrm{~Hz}, \mathrm{H}-19 \mathrm{~b})]$. Os espectros de RMN ${ }^{13} \mathrm{C}$ desacoplado e DEPT permitiram identificar um total de 30 sinais de átomos de carbono (6 não hidrogenados, 6 monoidrogenados, 10 diidrogenados e 6 triidrogenados). Dentre os quais foram observados valores de deslocamentos químicos para uma ligação dupla do tipo metileno terminal $[\delta 111,81$ (C-26) e $\delta$ 147,88 (C-25)], de carbonos oximetínicos $[\delta$ 79,24 (C-3) e $\delta 77,16(\mathrm{C}-24)]$ e de grupos metilas $(\delta 14,40 ; 17,61$; $18,43 ; 18,73 ; 19,72$ e 25,84$)$. As correlações observadas no espectro $\mathrm{HMBC}$ entre o sinal em $\delta 1,74(\mathrm{Me}-27) \mathrm{com}$ $\delta 77,16(\mathrm{C}-24)$ e do sinal em $\delta 4,04(\mathrm{H}-24) \operatorname{com} \delta 111,81$ (C-26) definiram a posição do grupo hidroxila em C-24. A presença no espectro de $\mathrm{RMN}{ }^{13} \mathrm{C}$ de sinais duplicados 


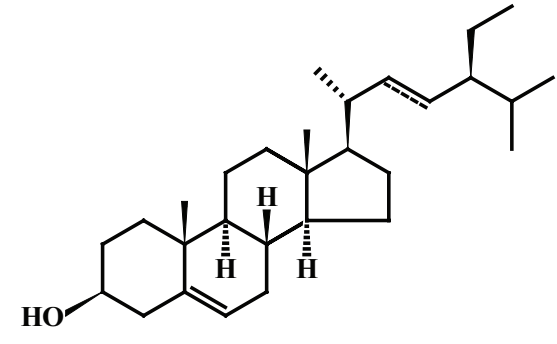

122,23 diidro $2 \Delta^{22,23}$

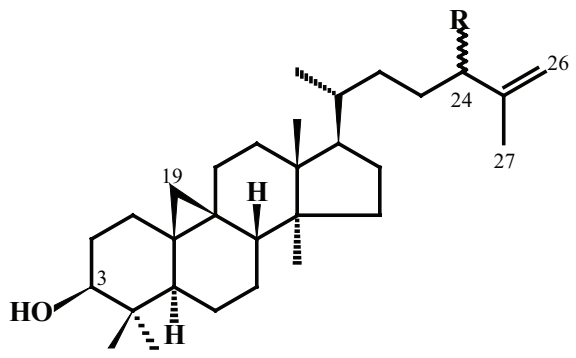

$$
\begin{aligned}
4 \mathrm{R} & =\alpha-\mathrm{OH} \\
5 \mathrm{R} & =\beta-\mathrm{OH}
\end{aligned}
$$

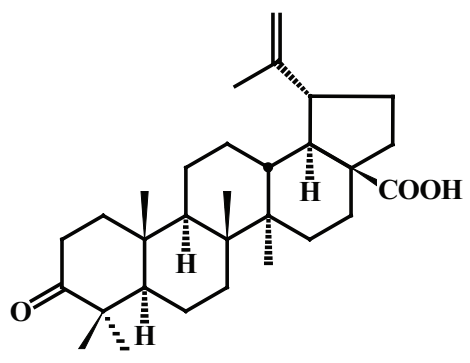

3<smiles>[R]c1cc(-c2oc3cc(OC)c(OC)c(O)c3c(=O)c2OC)ccc1Br</smiles>

$6 \mathrm{R}=\mathrm{R}_{1}=\mathrm{OMe}$

$7 \mathrm{R}=\mathrm{OMe}, \mathrm{R}_{1}=\mathrm{OH}$

$8 \mathrm{R}=\mathrm{OH}, \mathrm{R}_{1}=\mathrm{OMe}$

$9 \mathrm{R}=\mathrm{H}, \mathrm{R}_{1}=\mathrm{OH}$<smiles>CCO[C@H](c1ccc(O)cc1)[C@@H](O)[C@H](C)O</smiles>

11

atribuídos ao C-23 ( $\delta 31,89$ e $\delta 31,91), C-24(\delta 77,14$ e $\delta 77,16), \mathrm{C}-25$ ( $\delta 147,88$ e $\delta 147,90)$ e C-26 ( $\delta 111,81$ e $\delta 111,78)$ sugeriu a existência de uma mistura epimérica em C-24.

As estruturas dos flavonóides artemetina (6) (Martinez et al., 1987; Agrawal, 1989), crisosplenetina (7) (Sy; Brown, 1998), casticina (8) (Wang et al., 1989; Brown et al., 2003) e penduletina (9) (Wang et al., 1989) e tilirosídeo (11) (Kaouadji, 1990; Markham, 1993; Silva et al., 2005) foram identificadas pela comparação dos dados espectrais obtidos com os dos respectivos compostos descritos na literatura. A distinção entre as estruturas 7 e 8 foi feita com base na análise dos espectros COSY, NOESY e HMBC. Em relação à substância 7, correlações no espectro COSY entre o H-5' $[\delta$ 7,0 $(d$, $J=8,0 \mathrm{~Hz})]$, H-6' e H-2' [ $\delta$ 7,60-7,70 (m)] e destes últimos com o C-5' $(\delta$ 114,57), C-2' $(\delta 110,82)$ e C-6' $(\delta 122,53)$, respectivamente, foram observadas. No caso da substância 8, além das correlações no espectro COSY entre H-5' $(\delta 6,92)$ e H-6' $(\delta 7,60-7,70)$ e deste último com C-6' $(\delta 121,50)$, C-2' $(\delta 114,28)$ e C-2 ( $\delta 155,62)$, o espectro NOESY evidenciou a correlação espacial entre o H-5' $(\delta 6,92)$ com o grupo metoxila em C-4' $(\delta 3,92)$.

A substância 10 teve sua estrutura identificada com base na análise dos dados espectrais (IV e RMN, incluindo COSY, HMQC e HMBC). O espectro na região $\mathrm{IV}$, obtido em $\mathrm{KBr}$, revelou bandas de absorção indicativas de anel aromático (1609, 1517 e $\left.826 \mathrm{~cm}^{-1}\right)$, 
grupos hidroxilas $\left(3494,3239,1255\right.$ e $\left.1082 \mathrm{~cm}^{-1}\right)$ e grupos alquilas saturados $\left(2923,1462\right.$ e $\left.1374 \mathrm{~cm}^{-1}\right)$. A análise dos espectros de RMN ${ }^{13} \mathrm{C}$ (desacoplado, DEPT e HMQC) permitiu reconhecer um total de 9 sinais correspondendo a 11 átomos de carbono (2 não hidrogenados, 6 monoidrogenados, 1 diidrogenado e 2 triidrogenados). Os espectros de $\mathrm{RMN}$, em $\mathrm{CDCl}_{3}$, evidenciou sinais para um anel aromático do tipo 1,4-dissubstituído $\left[\delta_{\mathrm{H}} 6,86(d\right.$, $J=8,4 \mathrm{~Hz}, \mathrm{H}-3$ e H-5) e 7,20 ( $d, J=8,4 \mathrm{~Hz}, \mathrm{H}-2$ e H-6); $\delta_{\mathrm{C}} 115,54(\mathrm{C}-3$ e $\mathrm{C}-5), 129,10$ (C-2 e C-6), 131,20 (C-1) e 155,96 (C-4)], uma cadeia alifática saturada contendo três átomos de carbono $\left[\delta_{\mathrm{H}} 3,93(d, J=8,4 \mathrm{~Hz}, \mathrm{H}-7)\right.$, $3,84(\mathrm{~m}, \mathrm{H}-8)$ e $1,02(d, J=6,2 \mathrm{~Hz}, \mathrm{H}-9) ; \delta_{\mathrm{C}} 87,34(\mathrm{C}-7)$, $\delta 71,71(\mathrm{C}-8)$ e $\delta 18,09(\mathrm{C}-9)]$ e para um grupo etoxila $\left[\delta_{\mathrm{H}} 1,23\left(\mathrm{t}, J=7,0 \mathrm{~Hz}, \mathrm{Me}-2^{\prime}\right)\right.$ e $3,51-3,34\left(\mathrm{~m}, \mathrm{H}-1^{\prime}\right) ; \delta_{\mathrm{C}}$ 64,29 (C-1') e 15,45 (C-2')]. As correlações observadas no espectro HMBC, especialmente entre o sinal em $\delta 3,93$ $(\mathrm{H}-7) \operatorname{com} \delta 129,10$ (C-2 e C-6), $\delta 71,71$ (C-8) e $\delta 64,29$ (C-1') definiram a posição do grupo etoxila. O valor da constante de acoplamento de H-7 $(8,4 \mathrm{~Hz})$ sugeriu uma configuração treo para 10. O espectro bidimensional de correlação homonuclear (COSY) além de definir as interações spin-spin entre os átomos de hidrogênios permitiu também atribuir inequivocamente todos os deslocamentos químicos. A comparação com dados da literatura (Balboul et al., 1996) permitiu confirmar a estrutura do treo-7-etoxi-8-hidroxi-diidroanol. Esta substância foi isolada previamente das partes aéreas de Narvalina domingensis (Compositae) (Balboul et al., 1996).

\section{CONCLUSÃO}

O estudo fitoquímico com extratos das folhas e do caule de $C$. sellowii conduziu ao isolamento de fitoesteróides (sitosterol e estigmasterol), triterpenos (cicloart-25-en-3 $\beta, 24 \alpha$-diol, $\quad$ cicloart-25-en-3 $\beta, 24 \beta$ diol e ácido betulônico), flavonóides (artemetina, crisosplenetina, casticina, penduletina e tilirosídeo) e fenilpropanóide (7-etoxi-8-hidroxi-diidroanol). Embora estas substâncias ocorram com freqüência em plantas superiores, estas classes são compatíveis com o perfil químico esperado para o gênero Croton e estão sendo descritas pela primeira vez na espécie em estudo.

\section{AGRADECIMENTOS}

Os autores agradecem a FAPEAL, CNPq, MCT-IMSEAR e BNB-RENORBIO pelas bolsas e apoio financeiro; ao Prof. Dr. Edilberto R. Silveira e a Daniel Esdras de Andrade Uchoa, do Centro Nordestino de Aplicação e Uso de RMN da Universidade Federal do Ceará, e a Vicente Carlos Costa de Oliveira, do Laboratório de Tecnologia Farmacêutica da Universidade Federal da Paraíba, pelos espectros de RMN.

\section{REFERÊNCIAS}

Agrawal PK, Thakur RS, Bansal MC 1989. Flavonoids. In: Agrawal PK (ed.) Carbon-13 NMR of flavonoids. Studies in Organic Chemistry. Amsterdam: Elsevier.

Almeida ABA, Miotto AM, Nunes DS, Spadari-Bratifisch RC, Souza-Brito ARM 2002. Mechanism of antiulcerogenic activity of semi-synthetic crotonin obtained from Croton cajucara Benth. Rev Bras Farmacogn 12(Supl. 1): 105-110.

Anjaneyulu V, Babu JS, Babu BH, Havi K, Connoly JD 1993. Two D:A-Friedo-oleanane derivatives from Euphorbia tortilis. Phytochemistry 33: 647-649.

Balboul BAA, Ahmed AA, Otsuka H, Adams A 1996. 4Hydroxyphenylpropan-7,8-diols and derivatives from Narvalina domingensis. Phytochemistry 42: 1191-1193.

Barbosa-Filho JM, Vasconcelos THC, Alencar AA, Batista LM, Oliveira RAG, Guedes DN, Falcão HS, Moura MD, Diniz MFFM, Modesto-Filho J. 2005. Plants and their active constituents from South, Central, and North America with hypoglycemic activity. Rev Bras Farmacogn 15: 392-413.

Barbosa-Filho JM, Medeiros KCP, Diniz MFFM, Batista LM, Athayde-Filho PF, Silva MS, Cunha EVL, Almeida JRGS, Quintans-Júnior LJ 2006. Natural products inhibitors of the enzyme acetylcholinesterase. Rev Bras Farmacogn 16: 258-285

Bittner M, Silva M, Aqueveque P, Kufer J, Jakupovic J, Murillo $\mathrm{R}$, 1997. Alkaloids and other constituents from Croton chilensis. Bol Soc Chil Quim 42: 223-228.

Brown GD, Liang GY, Sy LK 2003. Terpenoids from the seeds of Artemisia annua. Phytochemistry 64: 303-323.

Cai Y, Chen ZP, Phillipson J 1993. Diterpenes from Croton lechleri. Phytochemistry 32: 755-760.

Chen ZP, Cai Y, Phillipson JD 1994. Studies on the antitumor, antibacterial, and wound-healing properties of Dragon's blood. Planta Med 60: 541-545.

Carpenter RC, Sotheeswaran S, Sultanbawa MUC, Ternai B 1980. C-13 NMR studies of some lupane and taraxerane triterpenes. Org Magn Res 14: 462-465.

Craveiro AA, Alencar JW, Matos FJA, Machado MIL 1990. The essential oil of Croton adenocalyx A.DC. $J$ Essent Oil Res 2: 145-146.

Falcão HS, Lima IO, Santos VL, Dantas HF, Diniz MFFM, Barbosa-Filho JM, Batista LM 2005. Review of the plants with anti-inflammatory activity studied in Brazil. Rev Bras Farmacogn 15: 381-391.

Farnsworth NR, Blomster RN, Messmer WM, King JC, Persinos GJ, Wilkes JD 1969. A phytochemical and biological review of the genus Croton. Lloydia 32: $1-28$.

Hedberg I, Hedbrerg O, Madati PJ, Mshigeni KE, Mshiu EN, Samuelsson G 1983. Inventory of plants used in traditional medicine in Tanzania. II. Plants of the families Dilleniaceae-Opiliaceae.JEthnopharmacol 9: 105-127.

Hernandez J, Delgado G 1992. Terpenoids from aerial parts of Croton draco. Fitoterapia 63: 377-378.

Kaouadji M 1990. Acylated and nonacylated kaempferol monoglycosides from Platanus acerifolia buds. Phytochemistry 29: 2295-2297.

Kumar Y, Haridasan K, Rao RR 1980. Ethnobotanical notes on 
certain medicinal plants among some Garo people around Balphakram Sanctuary in Meghalaya. Bull Bot Surv India 22: 161-165.

Lencina C, Pires VS, Gosmann G, Taketa ATC, Schenkel EP 2001. Tilirosídeo em Croton gnaphalii Ball. Rev Bras Farmacogn 11: 89-93.

Leôncio d'Albuquerque I, Delle Monache F, Ferrari F, Marini-Bettolo GB 1968. p-trans-1,2-Epoxy1-propyl)phenyl isovalerate (sellovicine B), an antibacterial substance from Croton sellowii. Gazz Chim Ital 98: 1189-1196.

Lemos TLG, Monte FJQ, Matos FJA, Alencar JW, Craveiro AA, Barbosa RCSB, Lima EO 1992. Chemical composition and antimicrobial activity of essential oils from Brazilian plants. Fitoterapia 63: 266-268.

Lewis WH, Elvin-Lewis MPF 1977. Medical Botany: Plants Affecting Man's Health. New York: WileyInterscience.

Macari PAT, Emerenciano VP, Ferreira ZMGS 1990. Identificação dos triterpenos de Miconia albicans Triana através de análise por microcomputador. Ouim Nova 13: 260-262.

Macari PAT, Sousa RR, Crespo MLL, Martins PA, Vieira JDD, Velosa AC, Fávero O, Gomes EPC 2002. Comparação entre os metais presentes em Croton floribundus Spreng. e Baccharis dracunculifolia DC. Rev Bras Farmacogn 12(Supl. 1): 76-77.

Macedo M, Ferreira AR 2004. Plantas medicinais usadas para tratamento dermatológicos, em comunidades da Bacia do Alto Paraguai, Mato Grosso. Rev Bras Farmacogn 14(Supl. 1): 40-44.

Mahato SB, Kundu AP 1994. ${ }^{13} \mathrm{C}$ NMR spectra of pentacyclic triterpenoids - A compilation and some salient features. Phytochemistry 37: 1517-1575.

Markham KR 1989. Flavones, flavonols and their glycosides. In: Methods in Plants Biochemistry. Dey PM \& Harborne JB (Org.) Londres: Academic Press.

Marques VLL 2001. Derivados dicromenoxantônicos $e$ terpenoídicos das raizes de Tovomita brasiliensis (Guttiferae). Maceió, 70p. Dissertação de Mestrado - Programa de Pós-Graduação em Química e Biotecnologia, Universidade Federal de Alagoas.

Martínez V, Barberá O, Parareda JS, Marco JÁ 1987. Phenolic and acetylenic metabolites from Artemisia assoana. Phytochemistry 26: 2619-2624.

Otshudi AL, Vercruysse A, Foriers A 2000. Contribution to the ethnobotanica, phytochemical and pharmacological studies of traditionally used medicinal plants in the treatment of dysentery and diarrhoea in Lomela area, Democratic Republic of Congo (DRC). $J$ Ethnopharmacol 71: 411-423.

Palmeira Júnior SF, Moura FS, Alves VL, Oliveira FM, Bento ES, Conserva LM, Andrade EHA 2004. Neutral components from hexane extracts of Croton sellowii. Flavour Frag J 19: 69-71.

Palmeira Júnior SF 2005. Contribuição ao conhecimento quimiotaxonômico da família Euphorbiaceae. Estudo químico de duas espécies do gênero Croton (C. sellowii Baill. e C. brasiliensis Muell. Arg.). Maceió, $317 \mathrm{p}$. Tese de Doutorado - Programa de Pós-Graduação em Química e Biotecnologia, Universidade Federal de Alagoas.

Piacente S, Belisario MA, Del Castillo H, Pizza C, De Feo V
1998. Croton ruizianus: Platelet proaggregating activity of two new pregnane glycosides. J Nat Prod 61: 318-322.

Pieters L, De Bruyne T, Mei G, Lemiere G, Van Den Berghe D, Vlietinck AJ 1992. In vitro and in vivo biological activity of South American Dragon's blood and its constituents. Planta Med 58: 582-583.

Pieters L, De Bruyne T, Claeys M, Vlietinck A, Calomme M, Van Den Berghe D 1993. Iisolation of a dihydrobenzofuran lignan from South American Dragon's blood (Croton spp.) as an inhibitor of cell proliferation. J Nat Prod 56: 899-906.

Pieters L, De Bruyne T, Van Poel B, Vingerhoets R, Totte J, Van Den Berghe D, Vlietinck A 1995. In vivo wound healing activity of Dragon's blood (Croton spp.), a traditional South American drug, and its constituents. Phytomedicine 2: 17-22.

Randau KP, Florêncio DC, Ferreira CP, Xavier HS 2004. Estudo farmacognóstico de Croton rhamnifolius H.B.K. e Croton rhamnifolioides Pax \& Hoffm. Rev Bras Farmacogn 14: 89-96.

Santos PML, Schripsema J, Kuster RM 2005. Flavonóides $O$-glicosilados de Croton campestris St. Hill. (Euphorbiaceae). Rev Bras Farmacogn 15: 321325.

Schultz ARH 1984. Introdução à botânica sistemática. Porto Alegre: Editora da Universidade. Sharaf M, Mansour RMA, Saleh NAN 1992. Exudate flavonoids from aerial parts of four Cleome species. Biochem Syst Ecol 20: 443-448.

Silva DA, Costa DA, Silva DF, Souza MFV, Agra MF, Medeiros IA, Barbosa-Filho JM, Braz-Filho R 2005. Flavonóides glicosilados de Herissantia tiubae (K. Schum) Brizicky (Malvaceae) e testes farmacológicos preliminares do canferol 3,7-di-O$\alpha$-L-ramnopiranosídeo. Rev Bras Farmacogn 15: 23-29.

Sy LK, Brown GD 1998. Three sesquiterpenes from Artemisia annua. Phytochemistry 48: 1207-1211.

Wang Y, Hamburger M, Gueho J, Hostettemann K 1989. Antimicrobial flavonoids from Psiadia trinervia and their methylated and acetylated derivatives. Phytochemistry 28: 2323-2327. 